\title{
Capacitação em álcool e outras drogas para profissionais da saúde e assistência social:
}

\author{
relato de experiência
}

\author{
Pedro Henrique Antunes da Costa ${ }^{(a)}$ \\ Daniela Cristina Belchior Mota ${ }^{(b)}$ \\ Erica Cruvinel ${ }^{(\mathrm{c})}$ \\ Fernando Santana de Paiva(d) \\ Henrique Pinto Gomide(e) \\ Isabel Cristina Weiss de Souza ${ }^{(f)}$ \\ Leonardo Fernandes Martins ${ }^{(\mathrm{g})}$ \\ Pollyanna Santos da Silveira(h) \\ Telmo Mota Ronzani ${ }^{(i)}$
}

\section{Introdução}

O uso de álcool e outras drogas tem sido problematizado em várias esferas da sociedade brasileira. As consequências do abuso destas substâncias são múltiplas e percebidas em vários setores. Por afetar tanto a saúde individual quanto a coletiva, este fenômeno exige uma abordagem que agregue prevenção, tratamento, organização de práticas e serviços assistenciais e formulação de políticas públicas específicas ${ }^{1}$.

Entretanto, no setor da saúde, a formação profissional para atuação com os problemas relacionados ao uso de drogas é deficitária, baseada no saber médico, enfocando a dependência e não priorizando a prevenção ${ }^{2-5}$. Esse descompasso entre a relevância da temática e a qualificação insuficiente denota a importância de propostas de formação profissional, fornecendo, aos usuários e familiares, cuidados adequados.

Além do campo da saúde, o abuso de drogas está entre os principais problemas identificados pelos profissionais do Sistema Único de Assistência Social (SUAS) ${ }^{6}$. Em função do avanço das políticas de assistência social e suas capacidades institucionais, os serviços do SUAS oportunizam a realização de abordagens preventivas, encaminhamentos de usuários para tratamento, bem como a assistência e o suporte a familiares ${ }^{6}$. Nesse sentido, é importante que os profissionais dos dispositivos socioassistenciais também estejam capacitados para atender às demandas relacionadas ao uso indevido de drogas.

Visando minimizar o panorama deficitário da formação de profissionais do Sistema Único de Saúde (SUS) e SUAS, e concretizar novas práticas, os Centros Regionais de Referência sobre Drogas (CRRs) são criados. Sua implantação é coordenada pela Secretaria Nacional de Políticas sobre Drogas (SENAD) por meio do edital no 002/2010/GSIPR/SENAD do Plano Integrado de Enfrentamento ao Crack e outras Drogas (PIEC), instituído pelo decreto 7.179 de 20 de maio de 2010. Após reformulação do PIEC, dando origem, em 2013, ao Programa "Crack, é possível vencer" (PCPV), os CRRs passam a ser uma das estratégias de prevenção deste Programa, juntamente com outras capacitações à distância na área.

Regidos por editais, os CRRs fornecem uma série de cursos de capacitação presencial para profissionais com atuação nas políticas sobre drogas. Por

\footnotetext{
ab,c,d,e,g,h,il) Departamento de Psicologia, Instituto de Ciências Humanas, Universidade Federal de Juiz de Fora. Centro de Referência em Pesquisa, Intervenção e Avaliação em Álcool e outras Drogas. Rua José Lourenço Kelmer, s/n, campus universitário, Bairro São Pedro. Juiz de Fora, MG, Brasil.36036-900. phantunes.costa@gmail. com; danibelmota@ yahoo.com.br; ecruvinel@yahoo.com. br; fernandosantana. paiva@yahoo.com.br; henriquepgomide@gmail. com; leomartinsjf@gmail. com; pollyannassilveira@ gmail.com; tm.ronzani@ gmail.com (bolsista de Produtividade CNPQ). (f) Departamento de Psicobiologia, Universidade Federal de São Paulo. São Paulo, SP, Brasil. isabel.weiss8@ gmail.com
} 
intermédio da capacitação destes atores sociais, espera-se contribuir para a materialização de novas práticas e processos de cuidado/trabalho. Assim, representam uma nova proposta e, ao mesmo tempo, a principal estratégia de formação presencial permanente das políticas e programas na área, agregando uma visão ampliada do problema enquanto questão predominantemente de saúde pública. Segundo o site do PCPV, são 45 CRRs implantados em todas as regiões brasileiras, vinculados a grupos de professores e pesquisadores de instituições públicas de Ensino Superior, com alta concentração na região Sudeste ${ }^{7}$.

Contudo, sabe-se que o processo de incorporação do conhecimento à prática profissional é complexo, indo além da mera transmissão da informação ou aquisição de conhecimentos técnicos. Apesar de a disponibilização de subsídios contextualizados contribuir para aumentar o senso de autoeficácia dos profissionais ao lidarem com os problemas do uso/abuso de drogas ${ }^{8}$, somente estes fatores não resultam diretamente em mudanças práticas ${ }^{9}$.

Para isso, postula-se a formação de recursos humanos a partir da educação permanente ${ }^{10-12}$, somados à pesquisa e prática clínica, tendo como horizonte uma educação que seja reflexiva, problematizadora e que vise a transformação da realidade social ${ }^{8}$. Espera-se a superação da primazia dos procedimentos nos serviços, respaldados pelo saber técnico, abrindo espaço, também, para reflexões sobre as práticas no cotidiano do trabalho $0^{10,11,13}$.

Nesse sentido, é necessário compreender e refletir criticamente sobre o papel dos CRRs no cenário de formação e atuação sobre a temática do uso de álcool e outras drogas. Assim como outras propostas de formação/capacitação governamentais, entende-se que o modelo representado pelos CRRs possa ter limitações e pontos positivos. Acredita-se que, por meio da explicitação e discussão de experiências dos CRRs, seja possível visualizar melhor estes fatores, de modo a fortalecer suas potencialidades e reverter ou minimizar seus obstáculos, contribuindo positivamente para o cenário de formação profissional na área.

A partir do explicitado acima, o presente artigo objetiva discutir aspectos da capacitação de profissionais do SUS e SUAS, por intermédio de um relato de experiência de um CRR do estado de Minas Gerais. Para isso, são descritos e analisados: o perfil dos profissionais, a estrutura, a abordagem pedagógica dos cursos, o impacto nas concepções sobre a temática e rotinas dos profissionais, bem como as potencialidades e dilemas a serem superados pelo modelo dos CRRs.

\section{Relato de experiência}

\section{Estrutura e perfil dos profissionais}

O CRR abrangeu um município de grande porte de Minas Gerais e municípios vizinhos. A equipe gerencial e pedagógica possuía: um coordenador geral, quatro coordenadores de curso, cinco monitores, uma secretária e 15 docentes de áreas diversas, com experiência prática e de pesquisa na rede de atenção aos usuários de drogas.

As capacitações foram desenvolvidas quinzenalmente, com oito horas/aula por encontro, totalizando sessenta horas. Os alunos, profissionais da rede pública (SUS e SUAS), foram alocados em quatro modalidades de curso - descritas abaixo - de acordo com seus perfis profissionais. Para receber certificação de conclusão dos cursos, foi estipulada uma frequência mínima de $75 \%$ e a apresentação de um projeto de intervenção nos serviços. Cabe ressaltar que os cursos e suas formatações (temáticas, ementas, cargas horárias etc.) foram estabelecidos pelo edital supracitado. A fim de assegurar a participação dos profissionais e englobar a realidade dos serviços, foram realizadas reuniões de pactuação com gestores da saúde e assistência social dos municípios de abrangência do CRR. Após acordos firmados, iniciou-se o processo de inscrição e matrícula dos alunos, respectivamente.

O curso "Gerenciamento de casos e reinserção social para profissionais da rede SUS e SUAS" foi o curso que recebeu o maior número de inscrições (354). Ao todo, setenta profissionais efetuaram a matrícula e 54 concluíram o curso, em sua maioria, assistentes sociais, psicólogos, enfermeiros e agentes sociais. 
Foram inscritos 69 profissionais no curso de "Aperfeiçoamento em crack e outras drogas para profissionais da Atenção Primária à Saúde (APS)". Devido ao não-preenchimento das vagas por médicos, viabilizou-se a participação de enfermeiros com atuação na APS. Entre o total de profissionais inscritos, 45 concluíram a sua matrícula, sendo que $70 \%$ eram médicos e $30 \%$ eram enfermeiros.

No curso "Atualização em atenção integral aos usuários de crack e outras drogas para profissionais de hospitais gerais", foram disponibilizadas sessenta vagas. Foram inscritos 109 profissionais. Dentre o total de inscritos, 67 efetuaram a matrícula, com 62 concluintes, sendo a maioria enfermeiros, psicólogos e assistentes sociais.

Já o curso "Aconselhamento motivacional e intervenção breve" contou com duas turmas, com sessenta vagas em ambas. A primeira turma contou com 62 inscritos (17 profissionais do SUS e 45 profissionais do SUAS) e 48 profissionais concluíram o curso. Na segunda turma, 67 profissionais se inscreveram (17 do SUS e cinquenta do SUAS), com 58 concluintes. Participaram das duas turmas: assistentes sociais, psicólogos, agentes comunitários de saúde, enfermeiros, dentre outros.

\section{Abordagem pedagógica}

\section{Pressuposto}

Por se tratar de um processo de formação, visando instrumentalizar e estimular o desenvolvimento de novas práticas de trabalho, a proposta pedagógica do presente CRR foi embasada nos princípios que orientam a educação permanente em saúde ${ }^{14}$. As ações objetivaram contribuir para os processos formativos por meio de práticas pedagógicas que abarcassem a organização dos serviços, identificando problemas cotidianos e construindo soluções para os mesmos ${ }^{14}$.

Buscou-se o desenvolvimento do poder de captação dos profissionais sobre suas compreensões de mundo, abordando suas relações com ele em contínuo movimento. A adoção dos ditames da Educação Permanente em Saúde foi fundamental às transformações das práticas cristalizadas e naturalizadas encontradas nos diferentes dispositivos da saúde e assistência social, sinalizando a necessidade de as pensarmos criticamente.

Assim, as estratégias pedagógicas do CRR foram norteadas pelos princípios abaixo:

1) O processo de construção do conhecimento deve ocorrer a partir da realidade concreta na qual os profissionais estão inseridos;

2) O processo de formação adota uma perspectiva problematizadora da realidade, buscando favorecer a conscientização do profissional em relação ao mundo e seu trabalho, afastando-se da mera transmissão de conteúdos;

3) Compreende-se os profissionais como atores ativos no processo de ensino-aprendizagem, enquanto o professor atua como um mediador dessa relação, guiando-se por uma postura crítica e dialógica.

4) Deve-se produzir conhecimento que estimule a construção de processos de ação em sintonia com a integralidade e intersetorialidade, tendo em vista a natureza multifatorial do uso de drogas.

\section{Estratégias pedagógicas}

Para fomentar a capacidade pedagógica do CRR, procurou-se proporcionar, aos profissionais, a aquisição de novos conhecimentos, redimensionando o problema por meio de análises críticas da realidade, e ampliando a capacidade de atuação ${ }^{15}$. Logo, foram delineadas as seguintes estratégias pedagógicas:

1) Análise da atuação profissional: identificação dos principais problemas e possibilidades nas práticas e serviços para abordagem aos usuários de álcool e outras drogas.

2) Formulação de propostas de ação: os profissionais dialogaram sobre soluções para o enfrentamento das necessidades identificadas, fomentando capacidades resolutivas. Como parte do processo de avaliação do aprendizado, eles apresentaram propostas de intervenção, compatibilizando os conteúdos abordados com suas realidades e serviços. 
3) Adequação à realidade institucional: foram considerados possíveis constrangimentos das realidades dos serviços para a adoção de novas práticas, questionando quais mudanças nos processos de trabalho eram viáveis e poderiam repercutir na sustentabilidade das ações.

4) Recursos de aprendizagem: além dos cursos de capacitação presencial, foi desenvolvida uma plataforma de ensino online, disponibilizando: informações, aulas, materiais, ambientes de interação, discussão e esclarecimento de dúvidas.

\section{Avaliação do trabalho}

A avaliação do CRR foi procedida continuamente, buscando-se compreender as consequências dos cursos e suas propostas nas concepções e rotinas dos profissionais, bem como levantar potencialidades e dilemas a serem superados pelo modelo dos CRRs. A observação participante foi uma importante estratégia, gerando relatórios sistemáticos, e sendo realizada durante a implementação dos cursos, juntamente com reuniões semanais de planejamento. Para compartilhar o trabalho executado e aprimorar as estratégias pedagógicas adotadas, a coordenação geral supervisionou os coordenadores dos cursos que monitoraram o corpo docente.

Como forma de aprofundar as compreensões sobre atitudes, crenças e percepções dos profissionais sobre o tema, assim como avaliar o impacto do CRR em suas formações e concepções, no início e no final de todos os cursos, oito grupos focais foram realizados. Estes grupos tiveram média de seis participantes de diferentes categorias profissionais, serviços e setores, selecionados intencionalmente a partir de sua assiduidade e envolvimento nas aulas e demais atividades. O material foi transcrito, sistematizado e analisado a partir da análise de conteúdo temática ${ }^{16}$. Após leitura do material, as categorias foram divididas em dois temas: "Potencialidades do CRR" e "Dilemas a serem superados", com as inferências baseadas na presença das categorias e referencial teórico.

\section{Resultados e discussão}

\section{Potencialidades do CRR}

Devido à alta procura por capacitação, e pelo decorrer dos cursos, observou-se que o abuso de drogas configura-se, para os profissionais, como um grave problema, atingindo várias pessoas e camadas populacionais. É uma questão que faz parte de suas incursões no âmbito das políticas de saúde e assistência social. São escassos os subsídios para a capacitação profissional e, consequentemente, para as ações voltadas à prevenção e assistência aos usuários de drogas ${ }^{17}$. $O$ aprendizado acontece por meio de treinamentos rápidos e desarticulados, pela experimentação na prática (tentativa e erro), ou por intermédio de esforços individuais autodidatas ${ }^{4,5}$.

A maioria dos profissionais mostrou-se motivada ao longo dos cursos, com expectativa de aumentar os conhecimentos e técnicas para lidar com usuários de drogas no seu cotidiano. Tal motivação pode ser percebida pela abertura dos profissionais, durante as aulas, para a aquisição de conhecimentos e a busca por recursos práticos para o desenvolvimento de intervenções junto aos usuários.

Em suma, os cursos possibilitaram reflexões mais ampliadas sobre a temática, enfocando não apenas serviços e práticas isolados, mas, também, o trabalho em rede, discussões sobre a gestão, dentre outros fatores. Para almejar a consolidação de transformações, as capacitações devem possibilitar mudanças nos processos de trabalho, visando a integração entre profissionais e setores ${ }^{15}$, além de estimularem a implementação de novas práticas no dia a dia dos serviços ${ }^{8}$.

Além disso, a aquisição de conhecimentos esclareceu diversos fatores relacionados ao uso e abuso de drogas, dando mais segurança para a realização das intervenções. O CRR serviu como 'desmistificador' de vários aspectos como, por exemplo, a modificação da visão acerca do usuário. Tal fator é fundamental para abordagens mais efetivas, auxiliando na reorientação da prática e para a adesão ao tratamento, rompendo com a cultura do preconceito. O profissional passa, também, a evitar individualizações do problema, tentando compreendê-lo a partir de um prisma abrangente ${ }^{18}$. 
O corpo docente e a estrutura pedagógica mostraram-se qualificados, atendendo às expectativas. O formato dos cursos e das aulas foi avaliado positivamente, agregando grupos heterogêneos de profissionais com diferentes vivências. Isso possibilitou a abertura de discussões e trocas de experiências entre os profissionais - que, nos seus cotidianos, aparecem desarticulados, sem integração no planejamento e compartilhamento das ações - e, também, uma maior noção da realidade, ampliando a visão sobre os problemas ${ }^{19}$.

Os fatores referentes à gestão e ao trabalho em rede também foram bem avaliados, situando o abuso de drogas como uma questão de saúde e não somente de segurança pública. Os profissionais puderam ampliar a discussão para a pluralidade de ações necessárias, reconhecendo o caráter multifacetado do abuso de substâncias e rompendo com o senso comum ${ }^{15}$. Os cursos foram avaliados como responsáveis por suscitar ou reforçar esta discussão e apresentar, aos profissionais, a importância da articulação/comunicação entre setores e serviços.

Dentro da proposta pedagógica, se destacaram os projetos de intervenção, onde os alunos formularam propostas para serem implementadas em seus locais de atuação. Estas propostas foram elaboradas em grupos e, posteriormente, apresentadas e discutidas juntamente com os demais integrantes da turma. Neste momento, diversos projetos foram estruturados com aplicabilidade nos serviços, propiciando reflexão dos papéis e envolvimentos nas ações com usuários de drogas.

Houve um novo dimensionamento da problemática, com os profissionais considerando a maior complexidade da questão e a importância da integração da rede assistencial. Dessa forma, o CRR contribuiu para que os profissionais se compreendam em uma rede que engloba os setores da saúde e assistência social, dentre outros, sendo atores-chave no processo de implementação de ações efetivas aos usuários de drogas ${ }^{17}$.

\section{O CRR como promotor de implementação de ações: dilemas a serem superados}

Apesar das potencialidades destacadas, dificuldades são percebidas na transposição da aquisição de conhecimentos para a implantação de novas práticas. Na tentativa de implementação do que foi aprendido, os profissionais esbarram na própria complexidade da problemática e na incapacidade de os serviços e rede lidarem adequadamente com essa amplitude ${ }^{20}$. Afirmam acreditar na prevenção e no tratamento, mas reconhecem que os problemas existentes e a falta de continuidade das ações obstaculizam o trabalho. Desse modo, somente a aquisição de conhecimentos não é capaz de superar a realidade ${ }^{8}$.

Os profissionais demonstram ver o problema de forma ampliada, mencionam a importância do trabalho em equipe, de se conscientizar e envolver a comunidade, sobretudo na reinserção social dos usuários, mas esbarram em como fazer isso. Apesar dos pontos positivos das discussões sobre os contextos de atenção, eles relatam ter pouco poder de decisão e atuação ${ }^{3}$. Assim, a qualificação de recursos humanos deve ser encarada em um contexto amplo, em que pesam a necessidade de aprimoramentos na estrutura e definição de processos de trabalho que considerem os problemas relacionados ao consumo de drogas ${ }^{10}$.

A rede assistencial é descrita como desintegrada e desarticulada, com dificuldade de suprir a demanda existente, gerando descontinuidade dos atendimentos e encaminhamentos para tratamento. Há desconhecimento acerca das formas de trabalho dos próprios serviços da rede, problemas no trabalho interdisciplinar e insuficiência de recursos ${ }^{19}$. Os profissionais mostraram-se sobrecarregados e, em alguns momentos, desmotivados, relatando, também, falta de autonomia ${ }^{5}$. Assim, as ações de prevenção acompanham a desarticulação da rede de atenção aos usuários de drogas, carecendo de maior integralidade e intersetorialidade, com o trabalho ainda organizado de forma fragmentada ${ }^{20,21}$.

A ausência de guias clínicos, que orientem os cuidados nos diferentes níveis de atenção e de acordo com os casos, foi constantemente mencionada. Entretanto, ao mesmo tempo em que os direcionamentos sobre "o que fazer" e "como fazer" são escassos, os profissionais relatam ter pouca autonomia para realizar o trabalho que gostariam, devido aos engessamentos, restrições dos serviços e das práticas, além da falta de condições/estrutura e problemas na rede.

A gestão aparece desconectada dos serviços e profissionais, sobretudo da APS, com discursos, muitas das vezes, não representando a realidade do problema. A efetividade da implementação de 
ações preventivas ao uso de álcool e outras drogas aparece associada ao engajamento dos gestores no processo de implementação de tais estratégias². Dessa forma, alguns questionamentos são pertinentes, como: Qual o papel da gestão na implementação das políticas públicas? Como os CRRs podem impactar nessas questões trabalhando, também, na aproximação entre gestão e profissionais?

Ademais, algumas características dos próprios CRRs dificultam a transformação do conhecimento em prática. É pressuposto do conceito de educação permanente que o aprendizado ocorra na prática $10,11,14$. Contudo, os cursos são propostos sem continuidade. Quando os alunos voltam para suas realidades, com conceitos adquiridos e ações formuladas, os cursos acabam, não acompanhando a implementação das intervenções e dificultando sua continuidade. Esse aspecto torna-se frequente nas capacitações governamentais, com atuações pontuais e sem supervisão, dificultando a incorporação de mudanças na prática ${ }^{12}$.

Outro ponto é a necessidade da inserção de outros setores, como a educação e segurança social. Para uma implementação efetiva das estratégias de prevenção, torna-se imprescindível a adoção de uma lógica de interação entre diversos setores, a partir da compreensão das demandas e necessidades sociais ${ }^{6}$. Além disso, deve-se garantir a participação efetiva de diferentes grupos sociais na identificação, formulação e implementação das ações. A educação e o âmbito escolar, especialmente na prevenção ao uso/abuso de drogas, podem favorecer a implantação de políticas públicas voltadas para os jovens, possibilitando que alunos, professores e comunidade assumam o papel de promotores de sua própria saúde ${ }^{22}$. Contudo, na formulação dos CRRs estes setores/dispositivos foram desconsiderados, nos fazendo questionar a intersetorialidade amplamente difundida nas políticas, mas insuficiente na implementação das ações.

Apesar da avaliação positiva da proposta pedagógica, foram questionados alguns momentos de ênfase teórica que, segundo os profissionais, apareceram descolados das realidades práticas dos serviços. Mesmo com a preocupação de aliar teoria e prática, algumas aulas não conseguiram essa aproximação do conteúdo teórico ao cotidiano dos serviços. Ademais, considerou-se insuficiente o tempo de discussão dos projetos de intervenção, em detrimento da sua relevância dentro da proposta do curso.

Nesse sentido, faz-se necessária a realização de exercícios de planejamento e avaliação constantes, visando potencializar as propostas dialógicas, explicitando os pressupostos teóricos, mas aliando-os ao "como fazer", e considerando contextos que não necessariamente sejam os propagados idealmente pelas políticas públicas. Contudo, não se deve transformar as propostas em receituários, confundindo este "como fazer" com algo pronto e acabado. Isto é significativo, tendo em conta a dificuldade dos profissionais, assim como os demais atores (gestores e acadêmicos), de realizarem avaliações constantes de suas práxis profissionais e não caírem em naturalizações e reproduções das práticas cotidianas.

Assim, os cursos devem ser oportunidades de conhecimento e de se repensar a ação profissional. As propostas de qualificação devem priorizar abordagens contextualizadas às realidades dos profissionais ${ }^{8}$, além de fortalecê-los para construírem novas estratégias de atuação e as articularem em suas rotinas. Isto poderá repercutir nas atitudes, possibilitando superar a perspectiva de que a única solução é transferir o problema para os serviços especializados, via encaminhamento.

Considerando o cenário assistencial obstaculizado, os CRRs, ao proporem ações a partir de contextos idealizados, acabam fazendo com que os profissionais percebam quão problemático é o cenário no qual se inserem, e fiquem desmotivados. Questionam-se dois pontos cruciais: 1) a necessidade de uma descentralização das ementas e conteúdos dos cursos, para que os CRRs possam adequá-los às características específicas de suas localidades e dos profissionais; e 2) a construção de alternativas conjuntas com os profissionais a partir da realidade em que estão inseridos.

São necessários direcionamentos aos cursos, não relativizando totalmente os conteúdos indispensáveis para a atuação no âmbito das drogas. Contudo, é imprescindível uma flexibilização das ementas para que não se desconsidere o conhecimento prático dos profissionais e suas realidades. São duas dicotomias: entre a ementa a priori e o estabelecimento de conteúdos a partir da realidade dos alunos; e entre o conhecimento teórico dos professores e o prático dos profissionais. Aproveitar o conhecimento de ambos os lados, estabelecendo relações de aprendizagem horizontais, é fundamental 
para a construção de práticas contextualizadas e impactantes nos serviços. Como apontam Souza e Ronzani ${ }^{8}$, a reflexão contínua sobre as práticas, proporcionada pela educação permanente, pode propiciar o contato com o desconforto e a disposição para produzir conceitos e alternativas de práticas transformadoras.

No contexto dos serviços, observou-se, também, uma centralidade das tomadas de decisões na equipe de saúde e gestão. Sabe-se que o fortalecimento da discussão junto à sociedade é imprescindível para a construção de políticas mais contextualizadas ${ }^{19}$. Entretanto, as políticas públicas e sua implementação, por meio da gestão e profissionais, acabam por considerar a população como agentes passivos e despreparados para o enfrentamento da questão, e para assumir a posição de protagonistas de suas próprias vidas, o que demanda, do Estado e profissionais, uma função educativa da sociedade 20 .

Ainda, os conhecimentos acadêmicos não podem continuar alienados dos serviços, na contramão do que hoje se espera numa ação formadora, que é produzir e disseminar conhecimentos que se sustentem no processo de trabalho ${ }^{11}$. Dessa forma, como o CRR poderia contribuir para a quebra desse monopólio entre gestão, academia e profissionais na formulação e implementação das políticas sobre drogas? Será que o CRR poderia, a partir da ampliação de suas ações, estabelecer agendas de diálogo com movimentos sociais, instituições de cuidado e o Estado, colaborando para a aproximação, e não polarização, de tais atores e grupos?

Deve-se pensar na articulação das ações de educação permanente e gestão dos serviços como forma de contribuir para mudanças no trabalho por meio das capacitações. Tais fatores auxiliam a qualificar a implementação das ações e possibilitam a construção de estratégias adequadas à realidade social, o que só poderá ser efetivado a partir da interação entre os diferentes atores que compõem este cenário das políticas públicas ${ }^{8}$.

Pensando o processo de educação voltado para a realidade, não é possível abarcar essa realidade sem considerar quem a constitui e é constituído por ela: a sociedade. Por ser um tema atravessado por inúmeros determinantes sociais, faz parte do processo inserir, estimular e propagar análises e intervenções que rumem nesta direção participativa, mesmo tendo clareza de que um curso não será capaz de abranger toda esta discussão.

Finalmente, visando facilitar a integração entre ações das políticas nos municípios, envolvendo gestores, profissionais e sociedade, propõe-se a realização frequente de momentos formais de diálogo e troca de experiências. As seguintes ações podem ser operacionalizadas: 1) oficinas abertas e com participação de diferentes atores; 2) promoção de encontros entre associações/serviços; e 3) elaboração de atos compartilhados entre profissionais, grupos sociais e gestão. Tais momentos teriam como funções: promover espaços de trocas de saberes e integrar a rede de atenção aos usuários de drogas e suas famílias, por meio do engajamento e enfrentamento coordenado.

\section{Considerações finais}

A implantação do presente $C R R$, a despeito das limitações apresentadas, possibilitou a qualificação de profissionais, gerando conhecimento para abordagens mais integrais e reflexivas da problemática. Os cursos possibilitaram troca de experiências, discussão e programação de ações de prevenção e cuidado em relação ao uso de drogas.

Contudo, deve-se questionar se, para o tipo de formação profissional desejada, o modelo de curso conteudista seria o mais adequado. Para isso, é necessário pensar os CRRs para além da prevenção e o cuidado ao usuário de drogas, abertos às demandas profissionais e sociais, e não tão limitados a ementas preestabelecidas verticalmente. O CRR não pode atuar somente como centro de capacitação e transmissão de conhecimentos, mas como centro de referência, o que requer reformulações sobre seu modelo, e uma posição mais bem definida e atuante dentro das políticas sobre drogas, garantindo continuidade nas ações e auxiliando no processo de readequação da rede e reformulação das práticas. 


\section{Colaboradores}

Os autores trabalharam juntos em todas as etapas de produção do manuscrito.

\section{Agradecimentos}

À Secretaria Nacional de Políticas sobre Drogas (SENAD), Conselho Nacional de Desenvolvimento Científico e Tecnológico (CNPQ), Coordenação de Aperfeiçoamento de Pessoal de Nível Superior (CAPES) e Fundação de Amparo à Pesquisa do estado de Minas Gerais (FAPEMIG).

\section{Referências}

1. Secretaria Nacional de Políticas sobre Drogas. Relatório brasileiro sobre drogas. Brasília (DF): SENAD; 2009.

2. Ronzani TM, Mota DCB, Souza ICW. Prevenção do uso de álcool na atenção primária em municípios do estado de Minas Gerais. Rev Saude Publica. 2009; 43(1):51-61.

3. Moretti-Pires RO, Corradi-Webster CM, Furtado EF. Consumo de álcool e atenção primária no interior da Amazônia: sobre a formação de médicos e enfermeiros para assistência integral. Rev Bras Educ Med. 2011; 35(2):219-28.

4. Vargas D, Duarte FAB. Enfermeiros dos centros de atenção psicossocial em álcool e drogas (Caps ad): a formação e a busca pelo conhecimento específico da área. Texto Contexto Enferm. 2011; 20(1):119-26.

5. Kanno NP, Bellodi PL, Tess BH. Profissionais da Estratégia Saúde da Família diante de demandas médico-sociais: dificuldades e estratégias de enfrentamento. Saude Soc. 2012; 21(4):884-94.

6. Tribunal de Contas da União. Sistema Nacional de Políticas Públicas sobre Drogas. Brasília (DF): TCU; 2012.

7. Observatório Crack, é Possível Vencer [Internet]. Brasília (DF): Ministério da Saúde; 2014 [acesso 20 Set 2014]. Disponível em: http://www.brasil.gov.br/observatoriocrack/ index.html.

8. Souza ICW, Ronzani TM. Álcool e drogas na atenção primária: avaliando estratégias de capacitação. Psicol Estud. 2012; 17(2):237-46.

9. Fixsen DL, Naoom SF, Blase KA, Friedman RM, Wallace F. Implementation research: a synthesis of the literature. Tampa: University of South Florida, Louis de la Parte Florida Mental Health Institute, The National Implementation Research Network; 2005.

10. Ceccim RB. Educação permanente em saúde: desafio ambicioso e necessário. Interface (Botucatu). 2005; 9(16):161-77.

11. Ceccim RB. Educação Permanente em Saúde: descentralização e disseminação de capacidade pedagógica na saúde. Cienc Saude Colet. 2005; 10(4):975-86.

12. Camargo Júnior KR, Campos EMS, Bustamante-Teixeira MT, Mascarenhas MTM, Mauad NM, Franco TB, et al. Avaliação da atenção básica pela ótica politico-institucional e da organização da atenção com ênfase na integralidade. Cad Saude Publica. 2008; 24 Supl 1:58-68.

13. Ceccim RB. Um sentido muito próximo ao que propõe a educação permanente em saúde. Interface (Botucatu). 2007; 11(22):343-63.

14. Ministério da Saúde. Política de educação e desenvolvimento para o SUS: caminhos para a educação permanente em saúde: polos de educação permanente em saúde. Brasília (DF): MS; 2004. 
15. Coelho HV. Escola de redutores de danos: experiência de formação na perspectiva da Saúde Coletiva. Saude Debate. 2013; 37(n esp):70-81.

16. Bardin L. Análise de conteúdo. Lisboa: Edições 70; 2010.

17. Barros MA, Pillon SC. Atitudes dos profissionais do Programa Saúde da Família diante do uso e abuso de drogas. Esc Anna Nery. 2007; 11(4):655-62.

18. Moraes M. O modelo de atenção integral à saúde para tratamento de problemas decorrentes do uso de álcool e outras drogas: percepções de usuários, acompanhantes e profissionais. Cienc Saude Colet. 2008; 13(1):121-33.

19. Schneider JF, Roos CM, Olschowsky A, Pinho LB, Camatta MW, Wetzel C. Atendimento a usuários de drogas na perspectiva dos profissionais da estratégia saúde da família. Texto Contexto Enferm. 2013; 22(3):654-61.

20. Paiva FS, Costa PHA, Ronzani TM. Fortalecendo redes sociais: desafios e possibilidades na prevenção ao uso de drogas na atenção primária à saúde. Aletheia. 2012; 37(1):57-72.

21. Alves VS. Modelos de atenção à saúde de usuários de álcool e outras drogas: discursos políticos, saberes e práticas. Cad Saude Publica. 2009; 25(11):2309-19.

22. Müller AC, Paul CL, Santos NIS. Prevenção às drogas nas escolas: uma experiência pensada a partir dos modelos de atenção em saúde. Estud Psicol. 2008; 25(4):607-16. 
Como alternativa ao panorama deficitário de formação profissional para atuação na área do uso de álcool e outras drogas, e visando à incorporação de mudanças práticas, são criados os Centros Regionais de Referência sobre Drogas (CRRs). Faz-se necessário compreender e refletir sobre o papel dos CRRs nos cenários de formação e atuação sobre a temática. A partir disso, o presente artigo pretende discutir aspectos sobre a capacitação de profissionais do Sistema Único de Saúde e Assistência Social, por meio do relato de experiência de um CRR de Minas Gerais. A implantação do presente CRR possibilitou a qualificação de profissionais em direção a abordagens integrais e reflexivas sobre a temática. Contudo, são necessárias reformulações sobre o seu modelo, com uma posição mais bem definida e atuante dentro das políticas sobre drogas, garantindo continuidade nas ações e auxiliando na reformulação das práticas.

Palavras-chave: Capacitação de recursos humanos em saúde. Educação profissional em saúde pública. Educação continuada. Formação de recursos humanos.

Training on alcohol and other drugs for health and social care professionals: report on experience

As an alternative to the deficient área of professional education for working on alcohol and other drug use in Brazil, and in order to incorporate changes in practices, Regional Reference Centers for Drugs (CRRs) have been created. Therefore, it is necessary to understand and reflect on the role of CRRs within the scenario of education and action in this field. This paper aimed to discuss aspects of the education process for professionals in the Brazilian Health System and Social Assistance System, through a report on experience from a CRR in the state of Minas Gerais, Brazil. The implementation of this CRR enabled education for professionals towards comprehensive and reflective approaches in this field. However, reformulations of the CRR model are needed, with a better-defined and more active position within drug policies, so a to ensure continuity of actions and aid in reformulation of practices.

Keywords: Human resources training on healthcare. Professional education on public healthcare. Inservice education. Human resources education.

\section{Capacitación sobre alcohol y otras drogas para profesionales de la salud y asistencia social: relato de experiencia}

Como alternativa al panorama deficitario de formación profesional para la actuación en el área del uso de alcohol y de otras drogas y con el objetivo de la incorporación de cambios prácticos, se crearon los Centros Regionales de Referencia sobre Drogas (CRRs). Por lo tanto, resulta necesario comprender y reflexionar sobre el papel de los CRRs en los escenarios de formación y actuación sobre la temática. A partir de esa base, este artículo tiene el objetivo de discutir aspectos sobre la capacitación de profesionales de los Sistemas Únicos de Salud y de Asistencia Social, por medio del relato de experiencia de un CRR del estado de Minas Gerais. La implantación del presente CRR posibilitó la calificación de profesionales en dirección para abordajes integrales y reflexivos sobre la temática. No obstante, son necesarias reformulaciones sobre su modelo, con una posición mejor definida y actuante dentro de las políticas sobre drogas, asegurando continuidad en las acciones y auxiliando en la reformulación de las prácticas.

Palabras clave: Capacitación de recursos humanos en salud. Educación profesional en salud pública. Educación continuada. Formación de recursos humanos. 